\title{
Amine catalysis for the organocatalytic diboration of challenging alkenes
}

\author{
Albert Farre, Kaline Soares, Rachel A. Briggs, Angelica Balanta, David M. Benoit, Amadeu Bonet*
}

\begin{abstract}
The generation of in situ $\mathrm{sp}^{2}-\mathrm{sp}^{3}$ diboron adducts has revolutionised the synthesis of organoboranes. Organocatalytic diboration reactions have represented a milestone in terms of unpredictable reactivity of these adducts. However, current methodologies have limitations in terms of substrate scope, selectivity and functional group tolerance. Here we report a new methodology based on the use of simple amines as catalyst. This methodology provides a completely selective transformation overcoming current substrate scope and functional/protecting group limitations. Mechanistic studies have been included in this report.
\end{abstract}

Organoboron compounds are widely used in the synthesis of new materials and new drug candidates owing to the versatility of boron moieties to serve as platform to access to major functional groups ${ }^{1}$ During the last few years, the generation of $\mathrm{sp}^{2}$ nucleophilic boron moieties by the addition of Lewis bases and diboranes (symmetrical and unsymmetrical) has raised a growing interest. This is due to their versatility, selectivity, unexpected reactivity and sustainability, when compared to transition metal-based methods. ${ }^{2-4}$ The potential of this method is well illustrated in the transition metal-free diboration reaction reported by Fernandez and co-workers, where a simple alkoxide was employed to catalyse the diboration reaction. ${ }^{2 e}$ This seminal report not only shows an unexpected reactivity to perform a full economically atomic reaction, which was restricted to expensive transition metals, but also opens the door to new and fascinating methods for the enantioselective approach of the reaction. ${ }^{2 e, 2 i, 2 n}$

Although, there are clear advantages in terms of sustainability, the reaction is limited to monoarene and mono- or disubstituted alkyl alkenes. As such substrates like stilbenes, dienes or trisubstituted alkenes cannot be employed and functionalised. ${ }^{5}$ Functional/protecting group compatibility is also limited to groups that can tolerate strong bases like alkoxides. Other alternative methods for the in situ activation of the diboron reagent, like carbene, ${ }^{2 a-c}$ organolithium ${ }^{2 k, 3 e}$, thioethers ${ }^{2 m}$, or fluoride $^{2 \mathrm{k}}$, do not improve the functional/protecting group compatibility, there are problems of selectivity and/or they can only diborate alkynes.

Here we report the use of amines for the activation of diboron reagents and their use in the diboration of alkenes. We envisage that the use of an alternative Lewis base, that is less nucleophilic than alkoxides, organolithiums, carbenes or fluorine, will not only expand functional/protecting group compatibility but may overcome current substrate limitations, access to new reactivity and open doors to new borylation asymmetric

[a] Mr. Albert Farre, Miss. Kaline Soarez, Miss. Rachel A. Briggs, Dr Angelica Balanta, Dr. David Benoit, Dr. Amadeu Bonet Department of Chemistry

University of Hull

Cottingham Road, HU6 7RX, Hull

E-mail: a.bonet@hull.ac.uk

Supporting information for this article is given via a link at the end of the paper. approaches.

We become interested in the formation of ate complexes with diboron reagents using alternative and less nucleophilic Lewis bases. Marder, Norman and co-workers demonstrated that a weak Lewis base, 4-picoline, is unable to interact with the bis(pinacolato)diboron $\left(\mathrm{B}_{2} \mathrm{pin}_{2}\right)$ but it does interact with the more Lewis acidic bis(catecholato)diboron $\left(\mathrm{B}_{2} \mathrm{Cat}_{2}\right)$. They described the first structurally-characterised neutral $\mathrm{sp}^{2}-\mathrm{sp}^{3}$ diboronic ester. ${ }^{6}$ The reaction of the diboron reagent with a strong Lewis base forms a stable $\mathrm{sp}^{2}-\mathrm{sp}^{3}$ ate complex. However, the use of a weak Lewis base, promotes an equilibrium in solution between the free diboronic ester and the $\mathrm{sp}^{2}-\mathrm{sp}^{3}$ ate complex. In the presence of an excess of weak base, it leads to the formation of the $\mathrm{sp}^{3}-\mathrm{sp}^{3}$ adduct.

Recently, Ingleson and co-workers showed that 4-picoline or DBN in the presence of $\mathrm{B}_{2} \mathrm{Cat}_{2}$ can promote a 1,1 ' rearrangement and this is highly dependent on the solvent. ${ }^{7}$ Both authors reported that use of an amine can cause the decomposition of $\mathrm{B}_{2} \mathrm{cat}_{2}$ forming $\mathrm{B}(\mathrm{cat})_{2}{ }^{-}$. Despite these seminal reports, these adducts have not been tested in organic transformations. Santos and co-workers synthesised and isolated a $\mathrm{sp}^{2}-\mathrm{sp}^{3}$ alkyl diboronic ester containing a dative $\mathrm{N}-\mathrm{B}$ bond which effectively polarised the B-B bond. This diborane is unreactive with organic substrates but it transmetallates into transition metals in the absence of a base. ${ }^{3 a}$,

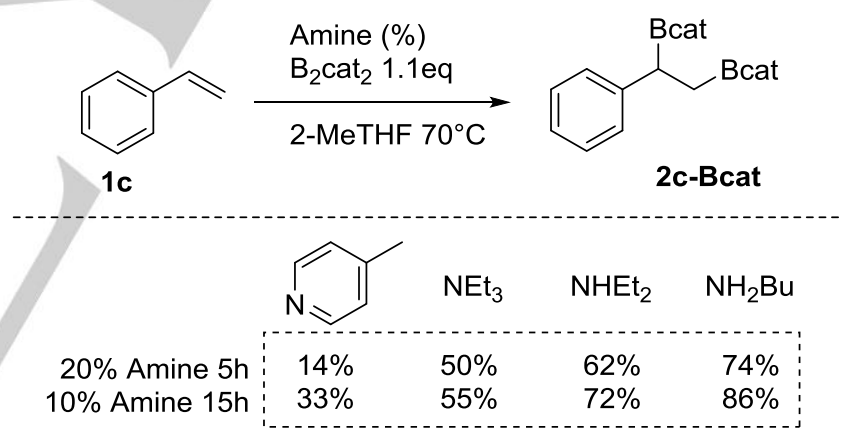

Scheme 1. Screening of different amines towards diboration reaction. NMR yields using 1,3,5-trimethoxybenzene as an internal standard. Conditions: substrate $(1.0 \mathrm{mmol})$, amine $(0.1 \mathrm{mmol}$ for $10 \%, 0.2 \mathrm{mmol}$ for $20 \%)$, B2cat 2 (1.1 mmol), 2-MeTHF $(4 \mathrm{~mL}), 70 \mathrm{C}$

We started our study by exploring the possibility of using an amine catalyst for the activation of $\mathrm{B}_{2} \mathrm{Cat}_{2}$ and its ability in performing a diboration reaction. We observed that primary amines provide higher yields than secondary, tertiary and aromatic possibly due to their increased Lewis basicity (Scheme 1). In contrast with the alkoxide method, the use of primary, secondary or tertiary Lewis bases does not show any problems of chemoselectivity and the hydroborated product has never been observed. We envisage that the use of a lower amount of amine could lead to higher yields because we are decreasing and postponing the formation of the $\mathrm{sp}^{3}-\mathrm{sp}^{3}$ adduct. Effectively, decreasing the amount of amine to $10 \mathrm{~mol} \%$, improved the reaction efficiency and $2 \mathrm{c}$-Bcat was obtained with $86 \%$ yield. We observed that solvent has a strong effect on the reactivity

This is the peer reviewed version of the following article: A. Farre, K. Soares, R. A. Briggs, A. Balanta, D. M. Benoit, A. Bonet, Chem. Eur. J. 2016, 22, 17552, which has been published in final form at http:// dx.doi.org/10.1002/chem.201603979. This article may be used for non-commercial purposes in accordance With Wiley Terms and Conditions for self-archiving. 
and 2-MeTHF and DCE provided higher yields. ${ }^{9}$ The use of $\mathrm{B}_{2} \mathrm{pin}_{2}$ or $\mathrm{B}_{2}$ neop $\mathrm{p}_{2}$ instead of $\mathrm{B}_{2} \mathrm{cat}_{2}$ resulted in no reactivity.

With the optimised reaction conditions in hand, we decided to explore the substrate scope of this novel diboration reaction. Styrene derivatives with different steric and electronic properties worked well providing $\mathbf{2 a - 2 h}$ in high yields (Scheme 2). We noticed that electron poor rings delivered higher yields than electron-rich ones providing mechanistic insights. The reaction can be easily scaled up with similar yields. Trisubstituted vinyl arenes and stilbenes are challenging substrates that go beyond the possibility of current methodologies. Pleasingly, when we expose trisubstituted vinyl arenes $\mathbf{1} \mathbf{i}, \mathbf{1} \mathbf{j}$ and stilbenes $(E$ and $Z$ ) $1 \mathbf{k}, 1 \mathbf{l}$ to our reaction conditions, diols $2 \mathbf{i}-2 \mathbf{l}$ were obtained in highyield and excellent diastereoselectivity.

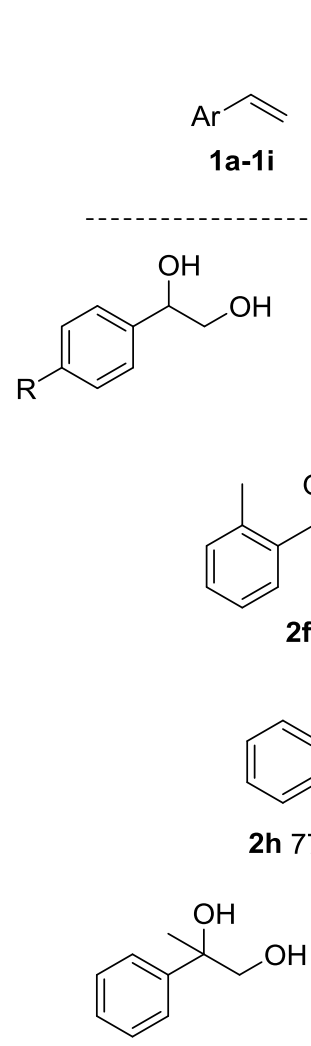

2j $69 \%$ dr $>99 \%$
1) $\mathrm{NH}_{2} \mathrm{Bu}(10 \%)$

$\mathrm{B}_{2} \mathrm{Cat}_{2} 1.1 \mathrm{eq}$

2-MeTHF, $70^{\circ} \mathrm{C}, 15 \mathrm{~h}$

2) $\mathrm{H}_{2} \mathrm{O}_{2} / \mathrm{NaOH}$

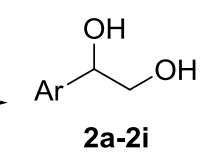

2a-2i diborated by current organocatalytic methodologies. Therefore overcoming this limitation is a major step in order to deliver a simple and broad methodology. Fortunately, in the optimized reaction conditions, trisubstituted alkylic olefins can be diborated with synthetically useful yields (Scheme $3, \mathbf{4 f}$ ). We were able to achieve the diboration of naturally occurring terpene 3-carene $\mathbf{3 g}$ with complete diastereoselectivity.

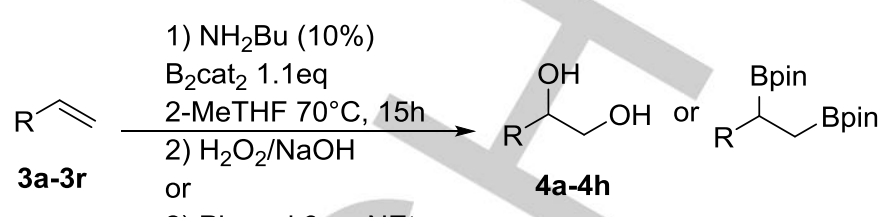

2) Pinacol 6eq, $\mathrm{NEt}_{3}$

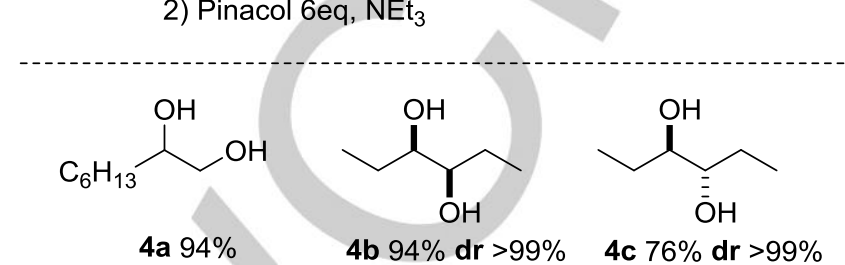

4a $94 \%$

4b $94 \%$ dr $>99 \%$

4c $76 \%$ dr $>99 \%$

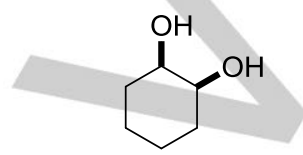

4d $77 \%$ dr $>99 \%$

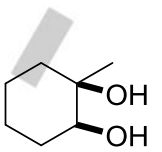

$4 f 70 \%$ dr $>99 \%$<smiles>O[C@@H]1C=C[C@H](O)CC1</smiles>

4e $36 \% 1,4$

$17 \% 1,2$<smiles>CC1(C)[C@H]2C[C@H](O)[C@](C)(O)C[C@@H]21</smiles>

4 g $60 \%$ dr $>99 \%$

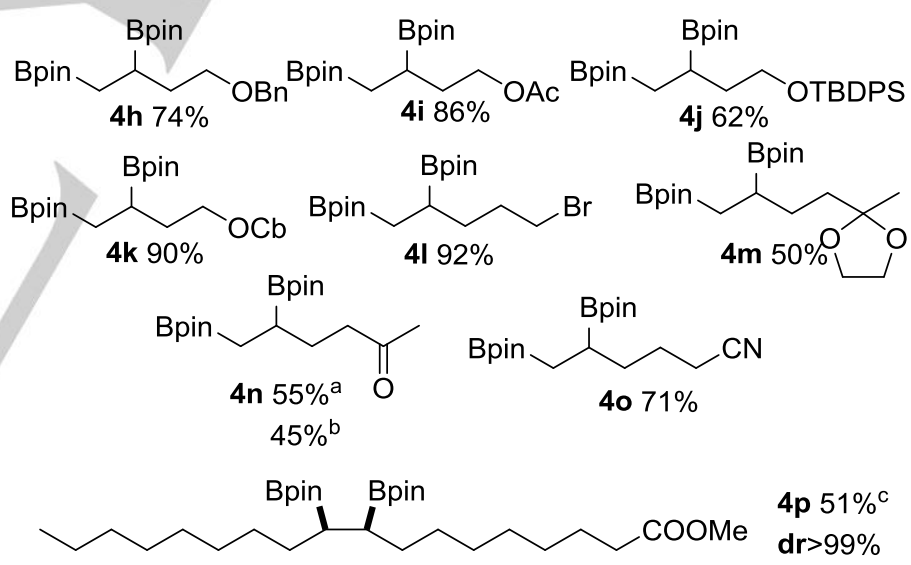

Scheme 3. Scope alkylic olefins. Diasterioselectivity evaluated by NMR. Isolated yields. Conditions: substrate $(1.0 \mathrm{mmol})$, amine $(0.1 \mathrm{mmol}), \mathrm{B}_{2}$ cat2 (1.1 mmol), 2-MeTHF (4mL), 70C, $15 \mathrm{~h}$ a NHEt 2 used instead of $\mathrm{NH}_{2} \mathrm{Bu}{ }^{\text {b }} \mathrm{NEt}_{3}$ used instead of $\mathrm{NH}_{2} \mathrm{Bu}$

The introduction of boron moieties using the in situ formation of $\mathrm{sp}^{2}-\mathrm{sp}^{3}$ diboron adducts has wide ranging applications and is rapidly expanding to new uses. ${ }^{4}$ Therefore, it is important to develop a general method which has a high functional and protecting group tolerance, we used our method with substrates containing the most common functional and protecting groups. a mixture of 1,4 and 1,2 addition products. Trisubstituted alkylic olefins are an important motive in nature and cannot be 
The catechol boronic ester moieties transform into an isolable pinacol boronic ester in a one-pot procedure, demonstrating the potential use of this method for the synthesis of highly functional building blocks.

We covered the most common alcohol protecting groups (Scheme 3, $\mathbf{3 h}-\mathbf{3 k}$ ). Acetate $\mathbf{3} \mathbf{j}$ and silicon protecting groups $\mathbf{3 k}$ are limitations of current organocatalytic borylations because the other methodologies lay with the use of alkoxides or fluorine anions and those can deprotect these groups. Our method successfully diborated both with vields up to $86 \%$. Carbamate $3 \mathbf{k}$ which could be easily further functionalized using lithiationborylation methodologies ${ }^{10}$, and bromine-containing $3 \mathrm{~m}$, which can be used as building block for coupling reactions provided almost complete transformation after the two consecutive reactions with vields up to $92 \%$. Acetal protecting groups are very common in synthetic sequences where ketones and aldehydes functionalities are present, and they are tolerated by our method as demonstrated by $3 \mathrm{~m}$. However, carbonyl functional groups could be used without protection. Ketone $3 \mathrm{n}$ could be transformed in the presence of secondary or tertiary amines with moderate yields. Primary amine remains unreactive probably due to the formation of the imine. Nitrile $\mathbf{3 0}$ can be borylated with synthetically useful yields. Methyl oleate 3p demonstrates that the reaction can be performed with fatty acidtype substrates with yields up to $51 \%$.

Although there is a clear synthetic advantage in our methodology there is still some substrate limitation. In our conditions, alkenes containing three phenyl substituents are unreactive and tetrasubstituted alkenes provide mostly the hydroborated product. The reaction is sensitive to alcohol containing substrates providing low yields, probably due to an exchange with the catechol group of the diboronic ester reducing their Lewis acidity.

\section{Mechanistic studies}

We performed NMR and computational experiments in order to gain an insight into the mechanism and the species involved in the reaction.

\section{NMR experiments}

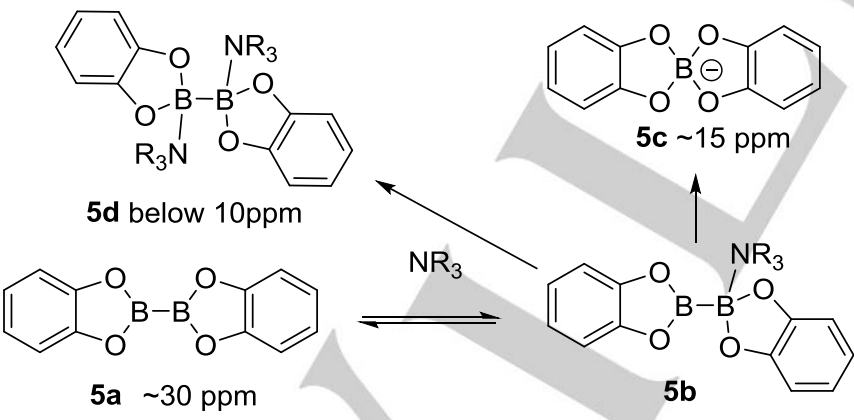

Scheme 4. Possible species in the ${ }^{11} B$ NMR for the mechanistic studies

The NMR experiments consisted of mixing each amine ( $n$ butylamine, diethylamine, triethylamine) with different amounts of $\mathrm{B}_{2} \mathrm{Cat}_{2}$. As expected, three main peaks in the ${ }^{11} \mathrm{~B}$ NMR were observed, which are in concordance with Marder and Ingleson. ${ }^{6,7}$

The most deshielded peak $(5 \mathbf{a}+5 \mathbf{b})$ consists of the equilibrium between the free $\mathrm{B}_{2} \mathrm{Cat}_{2}(5 \mathrm{a})$ and the $\mathrm{sp}^{2}-\mathrm{sp}^{3}$ adduct (5b). The chemical shift of the peak $(5 a+5 b)$ depends on: 1) the ratio of $5 b / 5 a$ at the equilibrium and 2 ) the polarisation of the $B$ $\mathrm{B}$ bond in $\mathbf{5 b}$. The peak at around $15 \mathrm{ppm}$ is the decomposition of the adduct to form $\left.\mathrm{B}(\mathrm{cat}){ }_{2}^{-}{ }^{-} \mathbf{( 5 c}\right)$ and the peak below $10 \mathrm{ppm}$ is the $\mathrm{sp}^{3}-\mathrm{sp}^{3}$ bisadduct $(\mathbf{5 d})$. If we keep constant the ratio of $\mathrm{B}_{2} \mathrm{Cat}_{2} / \mathrm{amine}$, we can evaluate how the $5 \mathbf{a}+5 \mathbf{b}$ peak shifts depending on the nature of the amines. The $\mathbf{5 a}+\mathbf{5 b}$ peak has a greater shift when the primary amine is used, followed by secondary and tertiary. If the chemical shift is related to the reactivity, these experiments could explain the reactivity. Marder and co-workers used as well solid ${ }^{11} \mathrm{~B}$ NMR experiments to obtain useful data for the description of similar species. ${ }^{6}$ We decided to study the $1: 1$ adducts with the different amines. In those experiments the same trend can be observed concluding that the primary amine provides a higher shift than secondary and tertiary amine. ${ }^{11}$

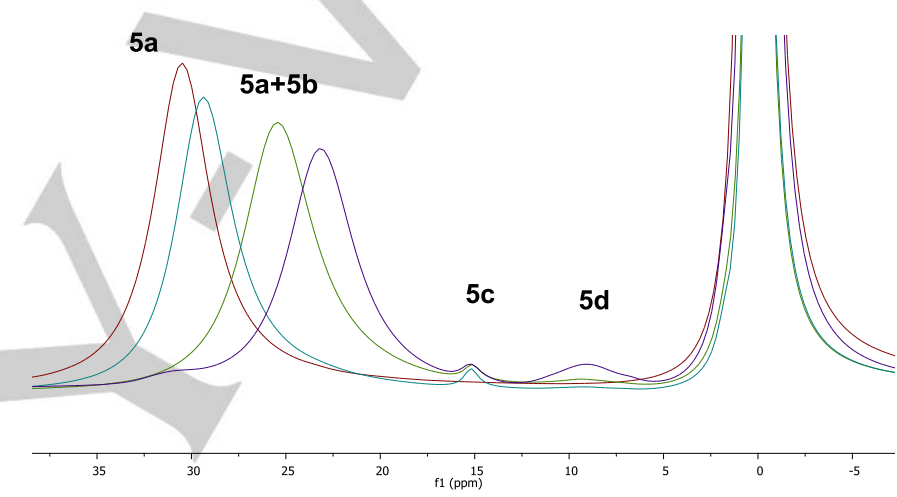

Figure 1. ${ }^{11} \mathrm{~B}$ NMR experiments $1 / 1$ ratio amine $B_{2} c_{2}(0.1 \mathrm{mmol}$ scale). Butylamine (purple), diethylamine (green), trimethylamine (blue) $\mathrm{B}_{2} \mathrm{cat}_{2}$ (red). External ${ }^{11} \mathrm{~B}$ NMR standard is used $\left(\mathrm{BF}_{3}-\mathrm{OEt}_{2}\right.$ signal at $\left.0 \mathrm{ppm}\right)$.

\section{Computational experiments}

We decided to perform preliminary gas-phase computational investigations to support the NMR data. We expected to gain mechanistic insights of the catalytic cycle and observe in which step of the cycle the substitution of the amine has an effect. Despite the fact that neglecting solvation effects might influence the height of the suggested energy barriers, we expect that the overall picture of the reaction remains valid.

We explore a mechanism for the organocatalytic diboration of olefins in which the amine activates the diboron reagent similarly to the mechanism described by Fernández and coworkers. ${ }^{2 e}$ The formation of $\mathbf{5 b}$ (Adduct $\mathbf{1 - S c h e m e ~ 5 ) ~ i s ~}$ favourable for all types of amines but the formation energy increases when going from primary to secondary amine and remains at around $-60 \mathrm{~kJ} / \mathrm{mol}$ for the tertiary amine. 
In contrast to the mechanism Fernández and co-workers reported, we only observed a single transition state (TS) for the addition. This could be due to the shallowness of the potential energy landscape or to the fact that in the study of Fernández and co-workers the catalyst was a methoxy group, which conferred a negative charge to the system. Our study uses amines as a catalyst and thus the overall system remains neutral.

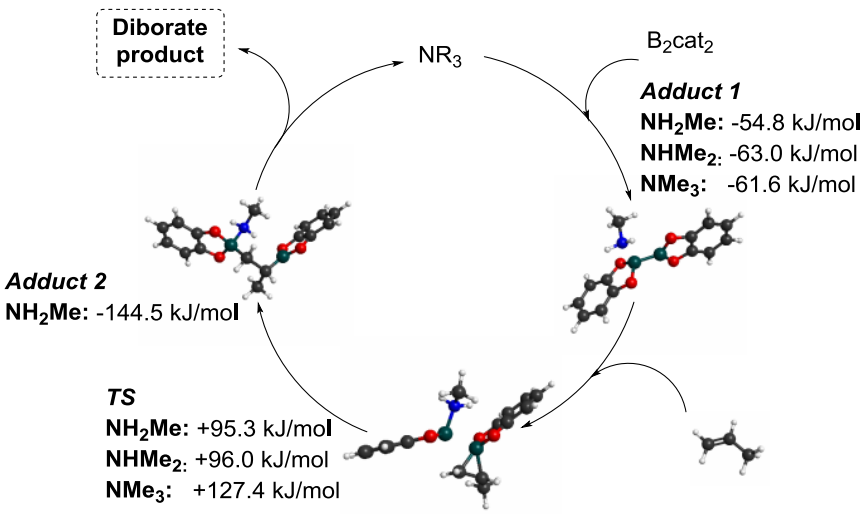

Scheme 5. Interaction energy of the fragments compared to the dissociated products at the RI-TPSS-D3/def2-TZVPP level of theory. This is equivalent to considering each step in the cycle as a separate reaction step. The energies in the scheme are those of Adduct 1 compared to $\mathrm{B}_{2} \mathrm{Cat}_{2}+\mathrm{NR}_{3}$ at infinite separation (Adduct 1); TS compared to Adduct 1 + olefin at infinite separation (TS); Adduct 2 compared to Adduct 1 + olefin at infinite separation (Adduct 2)

As can be seen from Scheme 5, the formation energy of Adduct 1 is favourable and similar for all amines with a slight preference for the secondary amine. However, the TS has a higher energy for the tertiary amine compared to the secondary and primary amines. This is likely caused by the increased steric bulk of the amine in this case and is in line with the decreased reactivity that we observe experimentally. The reaction then proceeds to form Adduct 2, in a predicted exothermic process $\left(-144.5 \mathrm{~kJ} / \mathrm{mol}\right.$ for $\left.\mathrm{NH}_{2} \mathrm{Me}\right)$ before regenerating the amine catalyst.

In summary, we have developed a simple method for the activation of diboronic esters and their use in diboration reactions. Our methodology relies in the use of amines as the catalyst and overcomes the many limitations of current methods in terms of both substrate scope and functional group tolerance. This method opens the door to the use of amines for chiral borylation reactions that are currently under study in our laboratory.

\section{Acknowledgements}

AB would like to thank the University of Hull for the starting grant which supported this research. AF would like to thank the EU for the Erasmus+ grant and University of Hull fellowship. KS would like to thank Brazil council for SwB (239091/2013-2) funding. We thank AllyChem for the gift of diboranes. Solid-State ${ }^{11} \mathrm{~B}$ NMR was obtained at EPSRC National Solid-state NMR Service at Durham. We acknowledge the VIPER high-performance computing facility of the University of Hull.

Keywords: organoboron $\cdot$ organocatalysis $\cdot$ Lewis base $•$ amines $\cdot$ density functional calculations

[1] a) Boronic Acids: Preparation and Applications in Organic Synthesis, Medicine and Materials, ed. D. G. Hall, Wiley-VCH, Weinheim, Germany, 2006.; b) Synthesis and Application of Organoboron Compounds, Ed. E. Fernandez, A. Whiting, Topics in Organometallic Chemistry 49, Spring, Switzerland, 2015; c) I. A. I. Mkhalid, J. H. Barnard, T. B. Marder, J. M. Murphy, J. F. Hartwig Chem. Rev., 2010, 110, 890-931; d) E. C. Neeve, S. J. Geier, I. A. I. Mkhalid, S. A Westcott T. B. Marder Chem. Rev.,2016, DOI: 10.1021/acs.chemrev.6b00193

[2] Symmetrical: a) K.-s. Lee, A. R. Zhugralin, A. H. Hoveyda, J. Am. Chem. Soc., 2009, 131, 7253-7255; b) K.-s. Lee, A. R. Zhugralin, A. H. Hoveyda, J. Am. Chem. Soc., 2010, 132, 12766-12766; c) C. Kleeberg, A. G. Crawford, A. S. Batsanov, P. Hodgkinson, D. C. Apperley, M. S Cheung, Z. Lin , T. B. Marder, J. Org. Chem., 2012, 77, 785-789. d) A. Bonet, H. Gulyás, E. Fernández Angew. Chem. Int. Ed., 2010, 49, 5130-5134; e) A. Bonet, C.Pubill-Ulldemolins, C.Bo, H. Gulyás, E. Fernández Angew. Chem. Int. Ed., 2011, 50, 7158-7161; f) A. Bonet, C. Solé, H. Gulyás, E. Fernández Org Biomol Chem. 2012, 10, 6621 6623 ; g) C. Pubill-Ulldemolins, A. Bonet, C. Bo, H. Gulyás, E. Fernández Chem. Eur. J., 2012, 18, 1121-1126; h) X. Sanz, G. M. Lee, C. Pubill-Ulldemolins, A. Bonet, H. Gulyás,S. A. Westcott, C. Bo, E. Fernández Org Biomol Chem. 2013, 11, 7004-7010; i) J. M. Zhang, H. H. Wu, J. L. Zhang, Eur. J. Org. Chem., 2013, 6263-6266; j) T. P. Blaisdell, T. C. Caya, L. Zhang, A. Sanz-Marco, J. P. Morken J. Am. Chem. Soc. 2014, 136, 9264-9267; k) S. Pietsch, E. C. Neeve, D. C. Apperley, R. Bertermann, F. Mo, D. Qu, M. S. Cheung, L. Dang, J. Wang, U. Radius, Z. Lin, C. Kleeberg T. B. Marder, Chem. Eur. J., 2015, 21, 7082-7098; I) J. Zheng, Y. Wang, Z. H. Li, H. Wang Chem. Commun., 2015, 51, 5505-5508; m) A. Yoshimura, Y. Takamachi, L. Han, A. Ogawa Chem. Eur.J. 2015, 21, $13930-13933$ n) N. Miralles, R. Alam, K. J. Szabó, E. Fernàndez Angew. Chem. Int. Ed. 2016, 55, $4303-4307$ o) L. Fang, L. Yan, F. Haeffner, J. P. Morken, J. Am. Chem. Soc. 2016, 138, 2508-2511; p) K. Yanga, Q. Song Green Chem., 2016, $18,932-936$

[3] Unsymmetrical: a) M. Gao, S.B. Thorpe, W. L. Santos, Org. Lett. 2009 11, 3478-3481 b) J. Cid, J. J. Carbó, E. Fernández Chem. Eur. J. 2014 20, 3616-3620; c) A. B. Cuenca, J. Cid, D. García-López, J. J. Carbó, E. Fernández; Org. Biomol. Chem., 2015,13, 9659-9664; d) N. Miralles, J. Cid, A. B Cuenca, J. J Carbó, E. Fernández Chem. Commun., 2015, 51,1693-1696; e) C. Kojima†, K. Lee, Z. Lin, M. Yamashita J. Am. Chem. Soc., 2016, 138, 6662-6669

[4] for a complete review of $\mathrm{sp}^{2}-\mathrm{sp}^{3}$ boryl chemistry: a) R. D. Dewhurst, E. C. Neeve, H. Braunschweig, T. B. Marder Chem. Commun., 2015,51, 9594-9607; b) J. Cid, H. Gulyás J. J. Carbó, E. Fernández Chem. Soc. Rev., 2012,41, 3558-3570

[5] See supporting information

[6] a) P. Nguyen, C. Dai, N. J. Taylor, W. P. Power, T. B. Marder, N. L. Pickett, N. C. Norman, Inorg. Chem., 1995, 34, 4290-4291 $\quad$ b) W. Clegg, C. Dai, F. J. Lawlor, T. B. Marder, P. Nguyen, N. C. Norman, N. 
L. Pickett, W. P. Power and A. J. Scott, J. Chem. Soc., Dalton Trans., 1997, 839-846;

[7] I. A. Cade, W. Y. Chau, I. Vitorica-Yrezabal, M. J. Ingleson, Dalton Trans., 2015, 44, 7506-7511

[8] a) M. Gao, S. B. Thorpe, C. Kleeberg, C. Slebodnik, T. B. Marder, W. L. Santos, J. Org. Chem., 2011, 76, 3997-4007, b) S. B. Thorpe, X. Guo, W. L. Santos, Chem. Commun., 2011,47, 424-426.

[9] See SI for full optimization of the reaction.

[10] Some representative examples of lithiation-borylation: a) J. L. Stymiest, V. Bagutski, R.M. French, V.K. Aggarwal, Nature, 2008, 456, 778-782, b) F. Schmidt, F. Keller, E. Vedrenne, V.K. Aggarwal, Angew. Chem. Int Ed., 2009, 48, 1149-1152, c) G. Dutheuil, M.P. Webster, P.A Worthington, V.K. Aggarwal, Angew. Chem. Int. Ed., 2009, 48, 63176319 , d) M. Althaus, A. Mahmood, J. Ramón Suárez, S.P. Thomas, V.K Aggarwal, J. Am. Chem. Soc., 2010, 132, 4045-4028, e) C. G. Watson, A. Balanta, T.G. Elford, S. Essafi, J. N. Harvey, V. K. Aggarwal J. Am Chem. Soc 2014, 136, 17370-17373, f) M. Burns, S. Essafi, J. R. Bame, S. P. Bull, M. P. Webster, S. Balieu, J. W. Dale, C. P. Butts, J. N. Harvey, V. K. Aggarwal Nature, 2014, 513, 183-188 


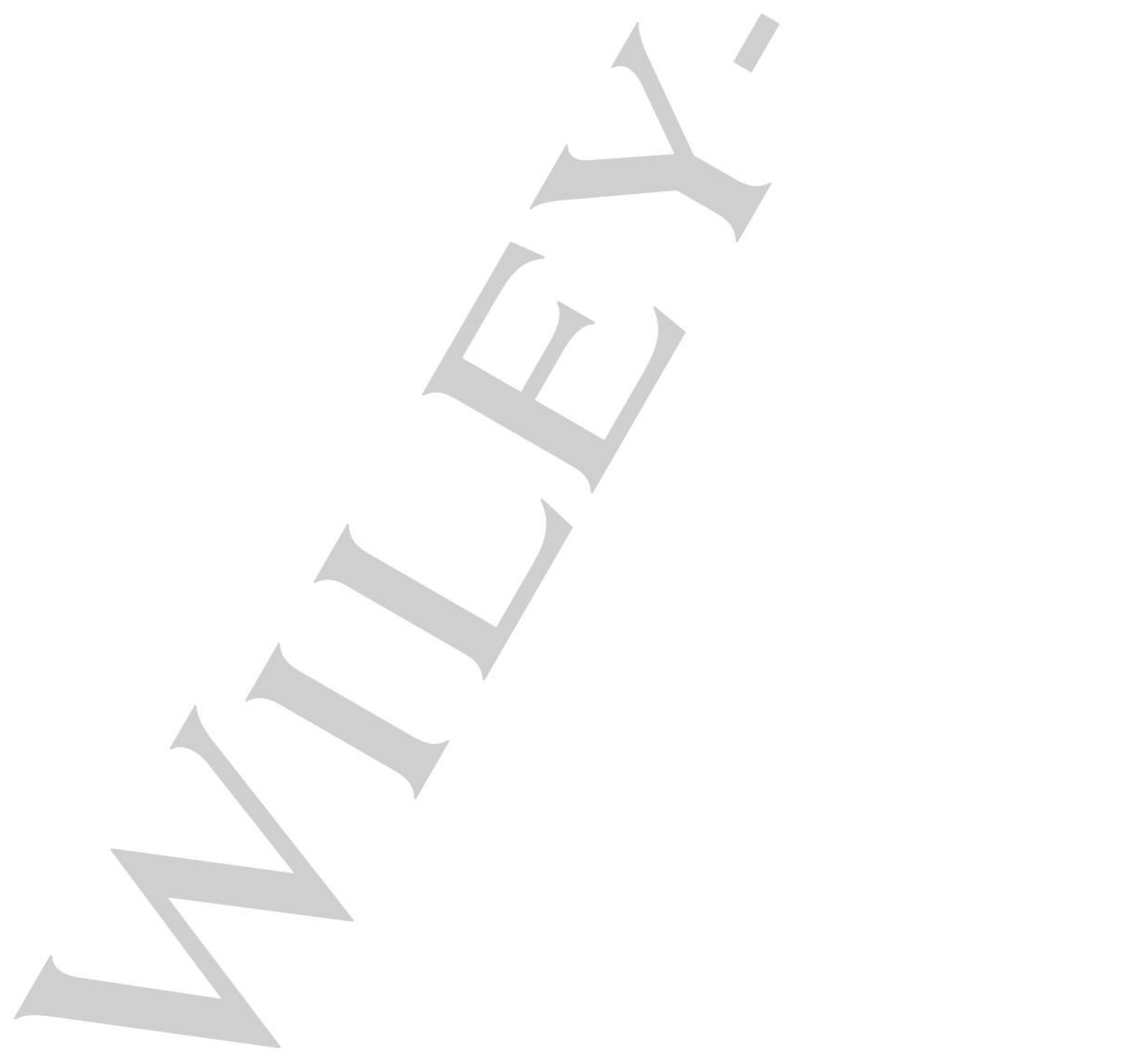

